\title{
ACCIón BIOLóGICA DE Aloe vera (Aloe barbadensis MILLER) SOBRE EL PERFIL LIPÍDICO EN RATAS ALBINAS wISTAR.
}

\author{
Enzio Foy Valencia ${ }^{1}$ \\ Vanessa Poquioma
}

\begin{abstract}
RESUMEN
Para demostrar el efecto hipolipemiante del consumo de extractos de Aloe vera (Aloe barbadensis Miller) al $20 \%$ se trabajó con lotes control y experimental de 10 ratas albinas para cada caso; tomando muestras sanguíneas basal al inicio. Luego de someterlas a una dieta hipergrasa y finalmente aplicarles la dieta experimental de Aloe vera al $20 \%$ se tomaron muestras sanguíneas y se pudo comprobar su acción reductora de lípidos séricos: los triglicéridos se redujeron en un 61.65\%; el colesterol en un $15.53 \%$; el LDL colesterol (colesterol malo) en $54.56 \%$ y se elevó el HDL(colesterol bueno) en $55.02 \%$
\end{abstract}

Palabras clave: Colesterol, Triglicéridos, HDL colesterol, Lipidemia.

\section{SUMMARY}

In order to demonstrate the lipid-lowering effect of the consumption of the extracts from Aloe vera (Aloe barbadensis Miller) at 20\%, we worked with control and experimental batches of albino rats for each case, taking baseline blood at the beginning. After the rats were on a high-fat diet and finally they were on an experimental Aloe vera diet at $20 \%$, blood samples were taken and it was possible to prove its reducing action of serum lipids: the triglycerides have been reduced by $61.65 \%$; the cholesterol by $15.53 \%$, the LDL cholesterol (bad cholesterol) by $54.56 \%$ and the HDL (good cholesterol) has been increased by $55.02 \%$.

Keywords: Cholesterol, Triglycerides, HDL cholesterol, lipid

\section{INTRODUCCIóN}

El aloe Aloe vera (Aloe barbadensis Miller) es una planta perteneciente a la familia botánica de las Liliaceas. Es rica en vitaminas, aminoácidos y enzimas. La palabra "aloe" deriva probablemente del árabe "alloeh", que significa "sustancia amarga" o bien del griego "Alos" que significa "mar". Viene designada con la palabra latina "vera" porque en la antigüedad esta variedad era considerada la más eficaz de las medicinas populares. Crece en climas tropicales, en terrenos arenosos y áridos. Se asemeja a un cactus pero en realidad es una planta perenne. Se caracteriza por hojas verdes y largas, duras, con forma de espada, con punta aguda y una serie de puntas de aspecto amenazador sobre cada borde. Las hojas crecen directamente del suelo, según un esquema de arandela. El género aloe tiene la capacidad de conservar el agua de lluvia lo que le permite sobrevivir por largos periodos de tiempo en condiciones de sequía. Después de tres años de vida de la planta, el gel contenido en las duras hojas verdes externas está al máximo de su contenido nutricional.

Se han efectuado estudios de los principios activos existentes en Aloe vera (Aloe barbadensis Miller), sin embargo, de la acción biológica de los principios referidos no hay mucha información $o$ investigaciones reportadas que verifiquen $o$ confirmen muchas de las virtudes que se le reconoce desde hace milenios.

\footnotetext{
${ }^{1}$ Universidad Ricardo Palma. Laboratorio de Bioquímica y Nutrición enfoval@hotmail.com
} 
Valdivia F., e Hidalgo M., sugieren que se hace necesario un estudio más exhaustivo de este tipo de plantas medicinales, para poder demostrar su utilidad en el manejo de la Diabetes Mellitus No Insulino Dependiente, por ejemplo; o de su acción hipolipemiante o hipocolesterolémica o su acción sobre el calcio y hierro séricos. Es necesario dar una explicación científica que justifique el uso empírico, en el Perú, de las plantas medicinales, con especial atención en Aloe vera (Aloe barbadensis Miller) a la cual se le atribuye efectos sobre calcio, hierro, lípidos, acción dermatológica.

La presente investigación de carácter experimental procura revalorizar el uso de Aloe vera (Aloe barbadensis Miller), pero como un producto al cual se le reconozca un carácter curativo o preventivo, que podría tener un efecto de carácter farmacológico que posibilitaría su aprovechamiento para el tratamiento de las lípidemias.. Es así que, al abordar de lleno la presente investigación, nos damos con que existen muy pocos trabajos que estén orientados hacia este propósito. Por lo que el presente trabajo se torna de suma importancia puesto que, de verificarse científicamente las propiedades del consumo de dietas suplementadas con extractos de Aloe vera (Aloe barbadensis Miller) con este producto estaríamos obteniendo un renovado valor terapéutico, haciéndolo, de esta forma, un insumo apreciado desde el punto de vista farmacológico y agrícola, así como desde una óptica sociológica puesto que el campesino que lo cultiva va a mejorar en su situación social y económica.

\section{MATERIAL Y MÉTODO}

\section{Material Biológico}

Muestras de las especies vegetales que proceden de los departamentos de Ancash y La Libertad.

\section{Procedimiento}

Para las especie a estudiar, se trabajó con planta seca y pulverizada, con la finalidad que sea administrada a los animales en experimentación ad libitum, de acuerdo a la metodología que indicamos.

\section{Metodología}

El método para llegar a los propósitos señalados consiste en lo siguiente:
1. Se adquirieron las muestras de Aloe vera (Aloe barbadensis Miller) directamente en las zonas de promoción del cultivo ecológico del producto que existen en la sierra norte de nuestro país; para luego proceder, en nuestros laboratorios a la elaboración de una dieta suplementada con extractos de Aloe vera (Aloe barbadensis Miller)

2. Se trabajó con dos bloques de especímenes de ratas albinas machos, uno para el control y otro para la aplicación experimental del producto a investigar, es decir, una dieta suplementada con extractos acuosos de Aloe vera (Aloe barbadensis Miller). Cada bloque consta de 10 ratas albinas. A cada uno se le hace un análisis bioquímico basal.

3. Con el bloque experimental de ratas albinas luego de aplicarles una dieta hipergrasa, por espacio de dos semanas, se efectuó un segundo análisis bioquímico del suero, luego delo cual se aplicó la dieta suplementada con Aloe vera (Aloe barbadensis Miller) igualmente por espacio de dos semanas. Finalmente se procedió nuevamente a determinar los valores séricos de lípidos en las ratas.

4. Los análisis bioquímicos del suero sanguíneo corresponden a las determinaciones del perfil lipídico.

\section{RESULTADOS}

Tabla 1. Variación de los niveles de Triglicéridos (mg/dl) en el período de 45 días

\begin{tabular}{|c|c|c|c|}
\hline \multirow{2}{*}{$\mathrm{N}^{\circ}$ Rata } & \multirow{2}{*}{$\begin{array}{c}\text { Dieta } \\
\text { Basal }\end{array}$} & $\begin{array}{c}\text { Día 15 } \\
\text { hieta }\end{array}$ & $\begin{array}{c}\text { Día 30 } \\
\text { Dieta } \\
\text { experimental }\end{array}$ \\
\hline 1 & 44.88 & 181.9 & 54.31 \\
\hline 2 & 42.08 & 179.7 & 65.68 \\
\hline 3 & 45.18 & 182.6 & 91.13 \\
\hline 4 & 41.78 & 179 & 85.23 \\
\hline 5 & 44.78 & 182 & 104.99 \\
\hline 6 & 42.18 & 179.6 & 135.45 \\
\hline 7 & 42.18 & 179.6 & 0 \\
\hline 8 & 44.83 & 182.15 & 64.77 \\
\hline 9 & 42.13 & 179.45 & 59.77 \\
\hline 10 & 45.14 & 182.4 & 32.04 \\
\hline Promedio & $\mathbf{4 3 . 5 1 6}$ & $\mathbf{1 8 0 . 8 4}$ & $\mathbf{6 9 . 3 3 7}$ \\
\hline D.S. & $\mathbf{1 . 4 5 4}$ & $\mathbf{1 . 3 9 3}$ & $\mathbf{3 5 . 9 4 4}$ \\
\hline
\end{tabular}


Cuadro 1. Valores Séricos de triglicéridos

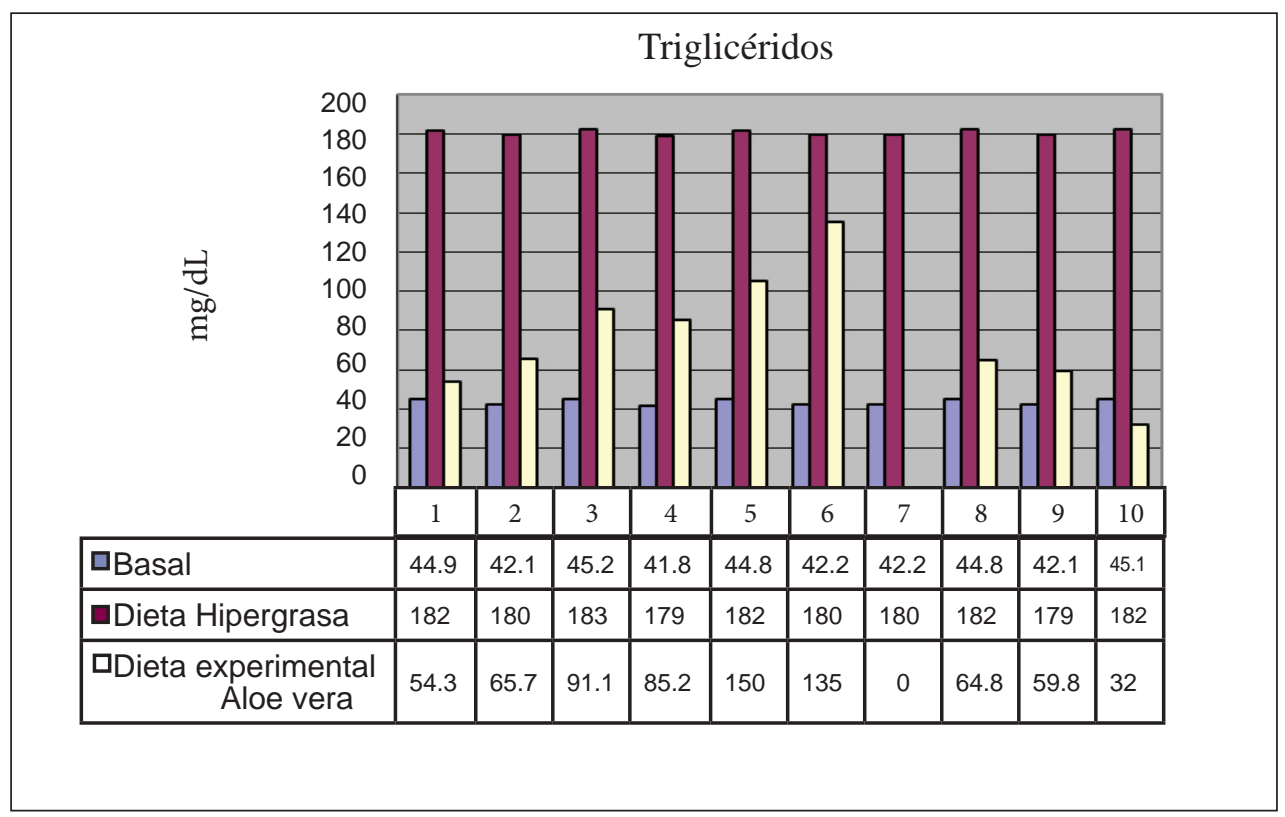

Cuadro 2. Valores séricos promedios de triglicéridos

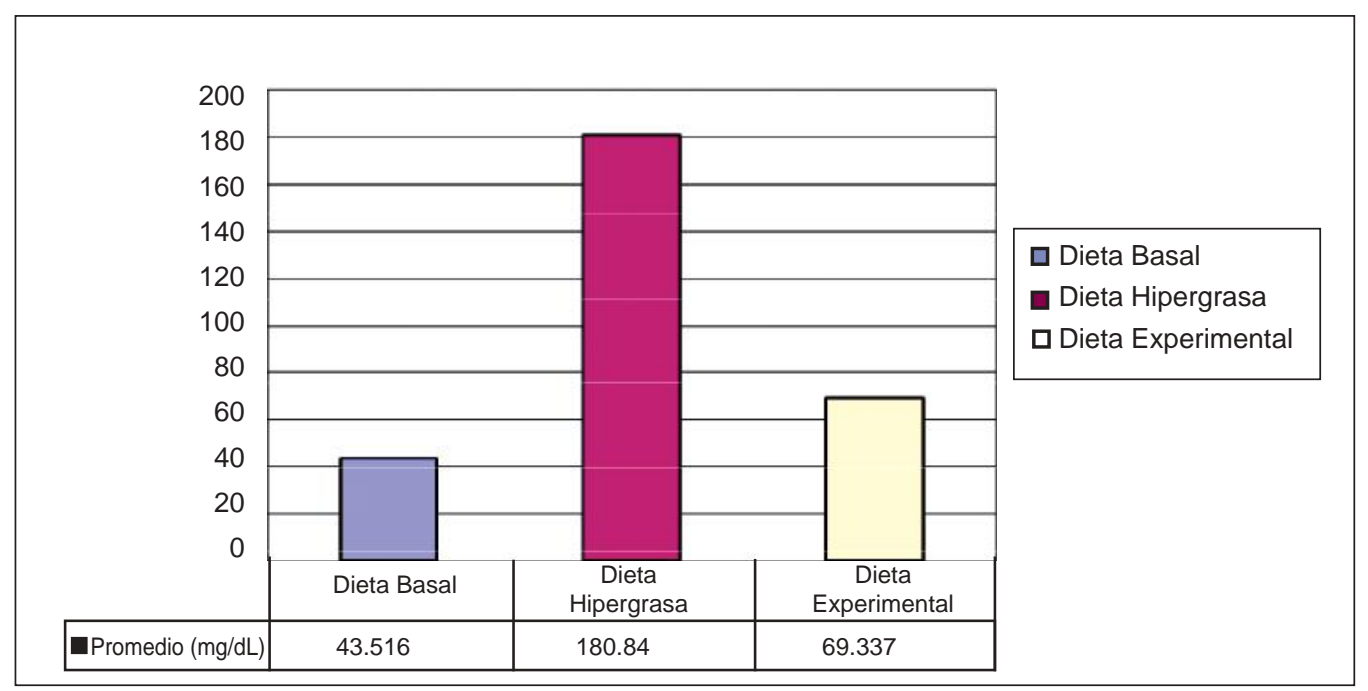


Tabla 2. Variación de los niveles de colesterol $\mathrm{mg} / \mathrm{dL}$ en el período de 45 días

\begin{tabular}{|c|c|c|c|}
\hline \multirow{2}{*}{$\mathbf{N}^{\circ}$ Rata } & \multirow{2}{*}{ Dieta Basal } & Día 15 & Día 30 \\
\cline { 3 - 4 } & & Dieta hipergrasa & $\begin{array}{c}\text { Dieta } \\
\text { experimental }\end{array}$ \\
\hline 1 & 38.01 & 97.76 & 82.03 \\
\hline 2 & 35.81 & 98.48 & 93.63 \\
\hline 3 & 38.71 & 95.56 & 122.2 \\
\hline 4 & 35.11 & 98.01 & 82.6 \\
\hline 5 & 38.11 & 94.86 & 86.56 \\
\hline 6 & 35.71 & 95.31 & 86.27 \\
\hline 7 & 35.71 & 95.31 & 0 \\
\hline 8 & 38.26 & 97.86 & 111.73 \\
\hline 9 & 35.56 & 98.26 & 80.05 \\
\hline 10 & 38.51 & 95.46 & 71.56 \\
\hline Promedio & $\mathbf{3 6 . 9 5}$ & $\mathbf{9 6 . 6 8 7}$ & $\mathbf{8 1 . 6 6 3}$ \\
\hline D.S. & $\mathbf{1 . 3 9 3}$ & $\mathbf{1 . 4 0 9}$ & $\mathbf{3 0 . 8 3 8}$ \\
\hline & & & \\
\hline
\end{tabular}

Cuadro 3. Valores Séricos de colesterol

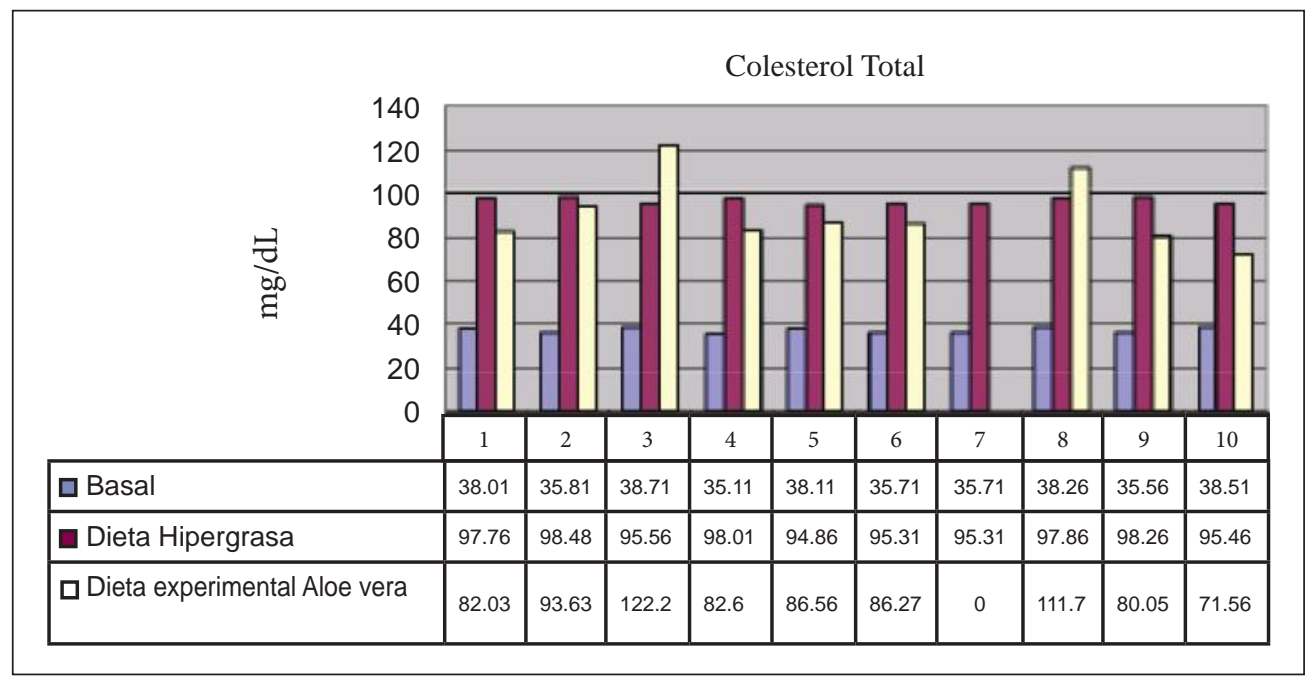

Cuadro 4. Valores séricos promedios de Colesterol Total

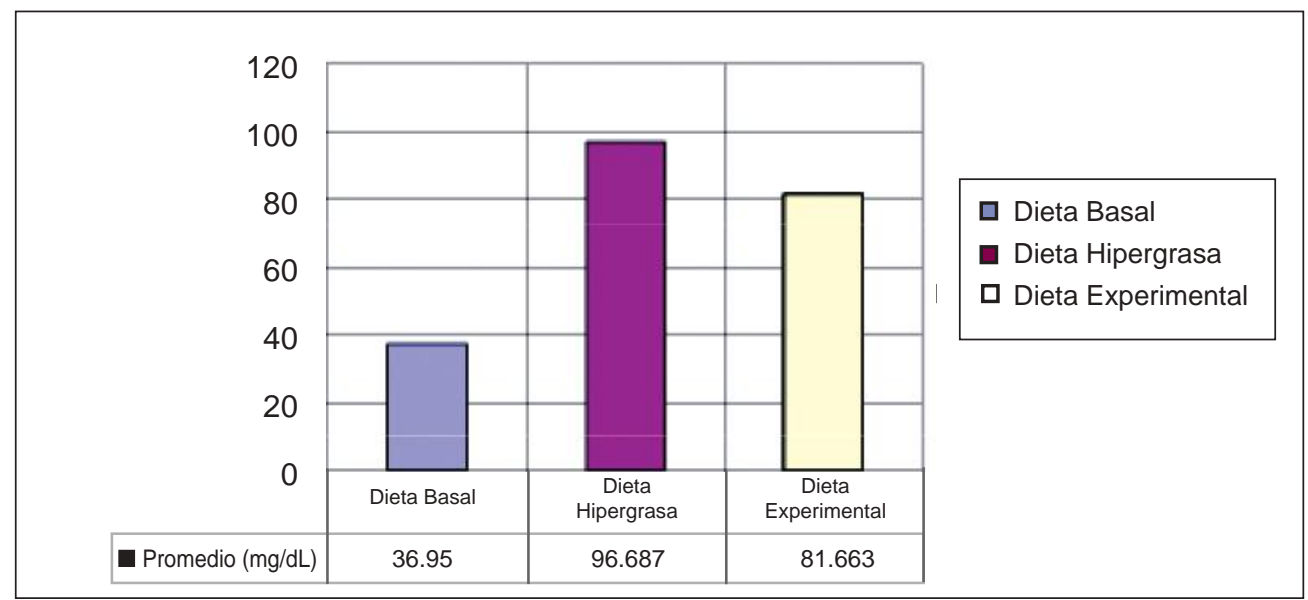


Tabla 3. Variación de los niveles de lipoproteínas de alta densidad (HDL) mg/dL (colesterol bueno en el período de 45 días)

\begin{tabular}{|c|c|c|c|}
\hline \multirow{2}{*}{$\mathbf{N}^{\circ}$ Rata } & \multirow{2}{*}{ Dieta Basal } & Día 15 & Día 30 \\
\cline { 3 - 4 } & & $\begin{array}{c}\text { Dieta } \\
\text { hipergrasa }\end{array}$ & $\begin{array}{c}\text { Dieta } \\
\text { experimental }\end{array}$ \\
\hline 1 & 26.07 & 37.98 & 41.46 \\
\hline 2 & 23.27 & 35.68 & 45.76 \\
\hline 3 & 26.37 & 35.78 & 124.9 \\
\hline 4 & 22.97 & 35.28 & 27.55 \\
\hline 5 & 25.97 & 38.68 & 59.41 \\
\hline 6 & 23.37 & 38.23 & 57.39 \\
\hline 7 & 23.37 & 38.23 & 33.88 \\
\hline 8 & 26.02 & 35.08 & 51.57 \\
\hline 9 & 23.32 & 35.53 & 72.56 \\
\hline 10 & 26.27 & 38.08 & 56.88 \\
\hline Promedio & $\mathbf{2 4 . 7}$ & $\mathbf{3 6 . 8 5 5}$ & $\mathbf{5 7 . 1 3 6}$ \\
\hline D.S. & $\mathbf{1 . 4 4 7}$ & $\mathbf{1 . 4 0 7}$ & $\mathbf{2 5 . 8 0 2}$ \\
\hline
\end{tabular}

Cuadro 5. Valores Séricos de HDL

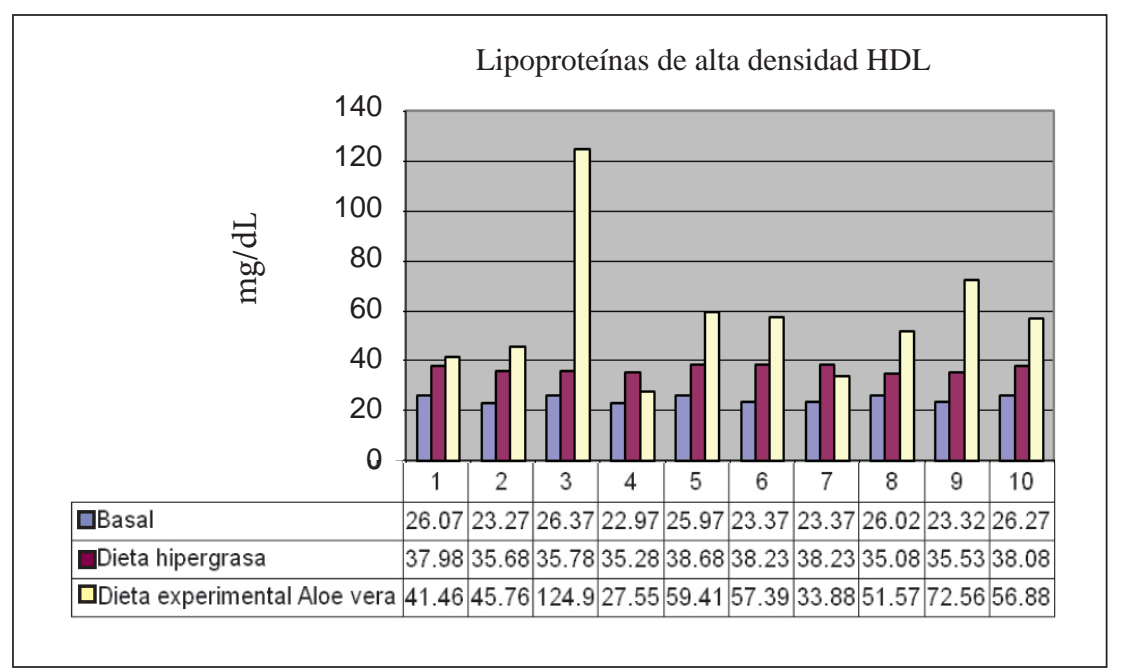

Cuadro 6. Valores séricos promedios de HDL

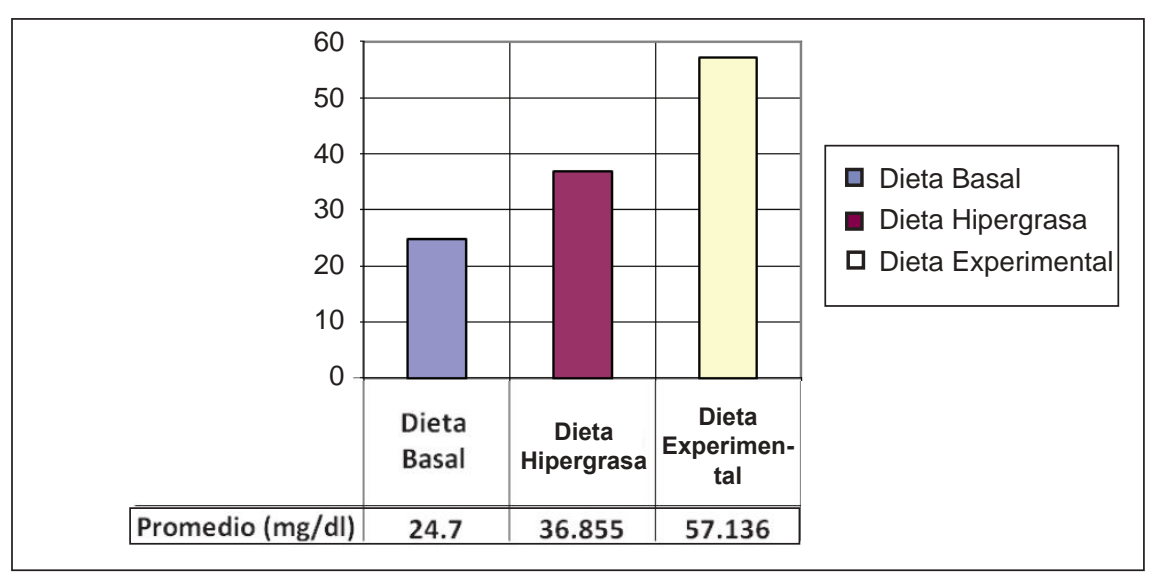




\section{Ecuación de Friedewald para la determinación de las lipoproteínas de baja densidad (LDL-C)}

LDL-C $(\mathrm{mg} / \mathrm{dL})=$ Colesterol total $(\mathrm{mg} / \mathrm{dL})-\mathrm{HDL}(\mathrm{mg} / \mathrm{dL})-\mathrm{VLDL}(\mathrm{mg} / \mathrm{dL})$

$\operatorname{VLDL}(\mathrm{mg} / \mathrm{dL})=$ Triglicéridos $/ 5$ (Cuando los Tg son menores de $400 \mathrm{mg} / \mathrm{dL})$

Tabla 4. Valores séricos de Lipoproteínas de baja densidad LDL- Colesterol según fórmula de Friedewald

\begin{tabular}{|l|c|c|c|}
\hline & Dieta Basal & $\begin{array}{c}\text { Dieta } \\
\text { hipergrasa }\end{array}$ & $\begin{array}{c}\text { Dieta } \\
\text { experimental }\end{array}$ \\
\hline $\begin{array}{l}\text { LDL-C } \\
(\mathbf{m g / d L})\end{array}$ & 3.5468 & 23.46 & 10.6596 \\
\hline
\end{tabular}

Cuadro 7. Valores séricos promedios de LDL-C

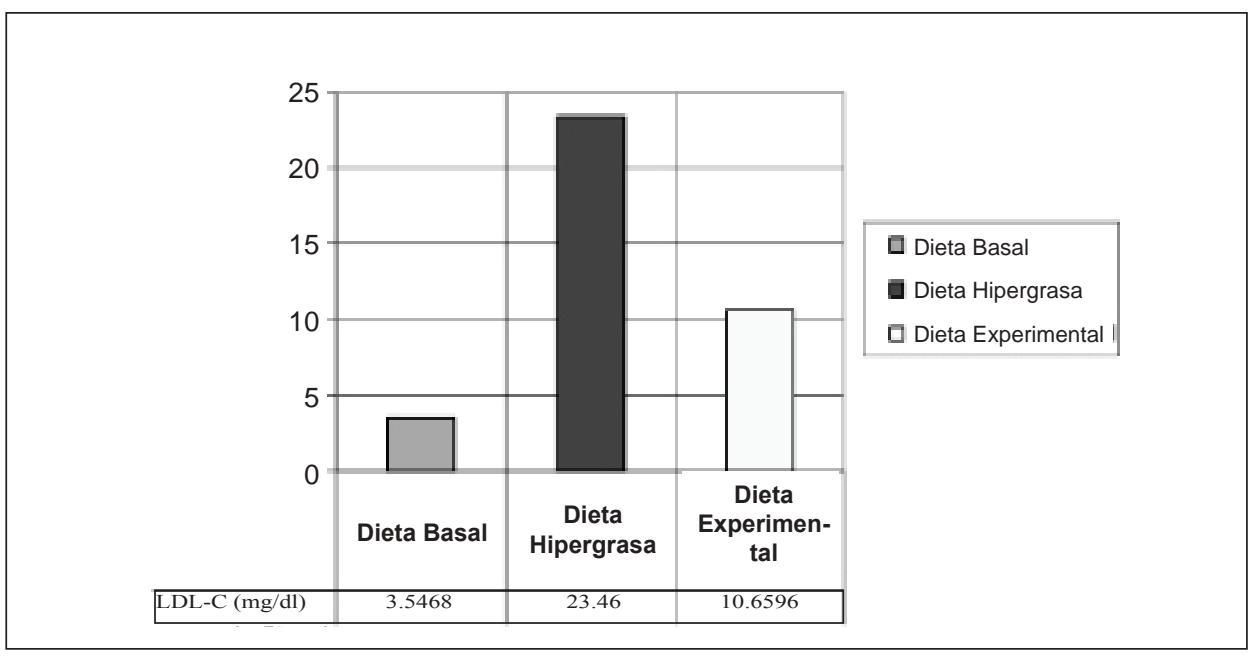

\section{DISCUSIóN}

Los triglicéridos sanguíneos se reducen notablemente $(61.65 \%)$ cuando se realiza la ingestión de una dieta la cual está suplementada con extractos de Aloe Vera, ingiriéndolos como componentes de la dieta en una proporción al $20 \%$. Considerando que los niveles de triglicéridos fueron inducidos a una elevación mediante la ingestión de una dieta excesiva de grasa (dieta hipergrasa), al incluirse en la dieta el extracto acuoso de Aloe vera los niveles de triglicéridos mejoraron, es decir bajaron. Si tenemos en cuenta que una elevación de los triglicéridos sanguíneos es perjudicial para la salud de las personas debemos de considerar que el consumo de el extracto acuoso de Aloe vera podría ayudar a una reducción de sus valores sanguíneos y por consiguiente a mejorar su salud con respecto a las concentraciones de lípidos.

Con respecto al colesterol total sanguíneo, éste es un componente de los lípidos corporales que cuando se elevan sus concentraciones sanguíneas trae problemas cardiovasculares y cerebro vasculares que pueden poner en peligro la salud de las personas así, como también su integridad; por consiguiente, el hecho que al haber inducido de forma experimental a una elevación del colesterol sanguíneo (hipercolesterolemia) en las ratas albinas durante 15 días, para luego hacerles un tratamiento con extracto acuoso de Aloe vera por 15 días también, permitieron una reducción bastante apreciable (15.53\%) de sus concentraciones sanguíneas. Por lo tanto, consideramos que si una 
persona que presenta elevaciones de su colesterol total, se somete a un tratamiento en el cual consuma como suplemento dietético extracto acuoso de Aloe vera podrá lograr una reducción y un mejoramiento de su colesterolemia.. Si tenemos en cuenta que cuando el colesterol se encuentra elevado se puede generar una ateroesclerosis (endurecimiento de las arterias y una posterior obstrucción arterial) que por lo general es de carácter irreversible; entonces consumiendo el extracto acuoso de Aloe vera se puede lograr una prevención y evitar que se generen las alteraciones referidas o en todo caso evitar que éstas se agraven en caso que ya existan.

Los lípidos o grasas son compuestos no solubles en agua, entonces para ser transportados en la sangre se forman unos compuestos denominados lipoproteínas que permiten transportarlos por la circulación general; éstas lipoproteínas pueden ser de diversos tipos según su densidad, una de ellas son las lipoproteínas de baja densidad (conocidas como LDL) que se caracteriza por presentar en su composición una alta concentración de colesterol que se va quedando en la circulación y en las arterias y que cuando sus concentraciones se elevan traen los problemas de endurecimiento de las arterias y sus consiguientes problemas cardiovasculares y cerebro vasculares; razón por la cual también se le conoce como el "colesterol malo". En el diseño experimental al proporcionarles a las ratas albinas la dieta hipergrasa durante 15 días se logró una elevación de estas lipoproteínas LDL ("colesterol malo"), y que cuando a los referidos animales de laboratorio, se les sometió al tratamiento con extracto acuoso de Aloe vera se logró una reducción del 54.56\%, lo cual consideramos que es un valor sorprendente en lo referente a una disminución del colesterol considerado como "malo" el cual es sumamente perjudicial para nuestro organismo. Como resultado de nuestro estudio estamos en condiciones de poder afirmar que el consumo de extracto acuoso de Aloe vera mejoran los niveles de las lipoproteínas de baja densidad LDL o "colesterol malo" y podrían servir para la prevención de los problemas circulatorios ocasionados por las elevaciones de estas fracciones de los lípidos sanguíneos.

Los resultados obtenidos se pueden proyectar hacia las personas en razón que el metabolismo de lípidos en las ratas albinas responde de manera similar a como sucede con los seres humanos, de allí que los trabajos experimentales de este tipo se suelen realizar con estos animales de laboratorio.

\section{CONCLUSIONES}

- El consumo de extractos de Aloe Vera en una proporción del $20 \%$ reduce los niveles de triglicéridos desde $180.84 \mathrm{mg} / \mathrm{dL}$ hasta $69.37 \mathrm{mg} /$ dL es decir un $61.65 \%$.

- El consumo de extractos de Aloe Vera en una proporción del $20 \%$ reduce los niveles de colesterol desde $96.68 \mathrm{mg} / \mathrm{dL}$ hasta $81.66 \mathrm{mg} / \mathrm{dL}$ lo cual significa una reducción del $15.53 \%$.

- El consumo de extracto de extractos de Aloe Vera en una proporción del $20 \%$ reduce los niveles de colesterol LDL (colesterol malo) desde23.46 $\mathrm{mg} / \mathrm{dL}$ hasta $10.69 \mathrm{mg} / \mathrm{dL}$, y representa una disminución del $54.56 \%$.

- El consumo de extracto de extractos de Aloe Vera en una proporción del $20 \%$ eleva los niveles de colesterol HDL (colesterol bueno) desde 36.85 $\mathrm{mg} / \mathrm{dL}$ hasta $57.13 \mathrm{mg} / \mathrm{dL}$, y representa una elevación del $55.02 \%$.

\section{REFERENCIA BIBLIOGRÁFICA}

1. Alarcón F. J.; Román R.; Flores J. L. 1993. Plantas medicinales usadas en el control de la diabetes mellitus. Ciencia, 44 (3), 363-381.

2. Valdivia F.; Hidalgo M. (1996). Uso de medicina tradicional en diabetes mellitus no insulinodependiente. Anales de la Facultad de Medicina UNMSM. Lima.

3. Capcha R.; Villena R. (1997) Determinación del efecto hipoglicemiante del fruto de Momordica charantia L. Tesis, Facultad de Farmacia y Bioquímica-UNMSM. Lima. Vol. 57(3); 180183.

4. Schwarz K.; Mertz W. (1957). A glucose tolerance factor and its differentiation from factor 3. Arch. Blochem Blophys 72:515-518.

5. Schwarz K.; Mertz W. (1959). Chromiun (IH) and the glucose tolerance factor. Arch Blochem Blophys 85:292-295

6. Mertz W. (1969) Chromium occurrence and function in biological systems. Physiol Rev. 49:163-239.

7. Mertz W. (1993) Chromium in human nutrition: a review. J. Nutr. 123:626-633 
8. Lock de Ugaz, O. (1998), Investigación Fitoquímica. Pontificia Universidad Católica del Perú. Fondo Editorial Lima.

9. Leopoldo Vásquez Núñez: Algunos Aspectos Actuales sobre el Uso de Plantas Medicinales en la Costa Peruana. Del Departamento de Ciencias Biomédicas de la Universidad Pedro Ruiz Gallo. Lambayeque-Perú.

10. Wust Walter. Guía de Especies Útiles de la Flora y Fauna Silvestre. Ediciones PEISA. 2003.

11. Brack Egg Antonio. Perú Diez Mil Años de Domesticación. Editorial Bruño. Lima 2003.

12. Non Shaw. Guía Ilustrada de Plantas Medicinales. Konemann Verlagsgesellschaft.

13. LIETTI Choleretic and cholesterol lowering properties of two artichoke extracts. Fitoterapia, 48, 153-158, 1977.

14. Lipid Digest (1994) International Lipid Information Bureau- Comité Peruano. Lima.

15. Cecílio Filho, A B. C Souza, R. J.; Braz, L. T.; Tavares, M. Curcuma: Planta medicinal, condimentar e de outros usos potenciais. Ciência Rural, Santa Maria, v. 30, (1), 171-175, 2000

16. Thapliyal, R. and Maru, G. B. Inhibition of cytochrome P450 isoenzymes by curcumins in vitro and in vivo Food Chemical Toxicology, v. 39 , n. 6, 541-547, 2001 .

17. Ihisge K.; Schubert, D.; Sagara, Y. Flavonoids protect neuronal cells from oxidative stress by three distint mechanism. Free Radic. Biol. Med 2001: 30(4):433-446.

18. Sood, A.; Mathew, R.; Trachtman, H. Cytoprotective effect of curcumin in human proximal tubule epithelial cells exposed to shiga toxin. Biochem Biophys Res Commun 2001:283:36-41.

19. Langlands, K.; Down, G. A.; Kealey, Tl. ID proteins are dynamically expresse in normal epidermis and dysregulated in squamous cell carcinoma. Cancer Res 2000: 60: 5929-5933.

20. Shuang-En Chuang, Cheng-Ann-Lii JenKun, Lin JK, Kuo Min-Liang. Inhibition by curcumin of diethylnitrosamineinduced hepatic hyperplasia, inflammation, cellular gene products and cell cycle-related proteins in rats. Food and Chemical toxicology 2000; 38(11):991-995.

21. Akira Asai, Life sciences, 67, 2785-2793, 2000)

22. I, Oshima H. Curcumin, an anti-tumor promoter and anti-inflammatory agent, inhibits induction of nitric oxide synthase in activated macrophages. Biochemical and Biophysical Research Communications 1995; 206:533-540.

23. AGARWAL GP: Prevention of atheromatous heart disease. Angiology 36: 485-492, 1985.

24. AL-AWADI FM, GUMAN KA: Studies on the activity of individual plants of an anti-diabetic plant mixture. Acta Diabetol Lat 24:37-42, 1987.

25. BLITZ JJ, SMITH JW, GERARD JR: Aloe Vera Gel in peptic ulcer therapy, preliminary report, $\mathrm{J}$ Am Osteopath Assoc 62:731-735, 1963.

26. BUNYAPRAPHATSARA N, YONGCHAIYUDHA S, RUNGPITARANGSI V, CHOKECHAIJAROENPORN O, Antidiabetic activity of Aloe Vera L. juice II. Clinical trial in diabetes mellitus patients in combination with glibenclamide. Phytomed 3:245-248, 1996.

27. DAVIS RH, DONATO JJ, HARTMAN GM, HAAS RC: Anti-inflammatory and wound healing activity of a growth abstance in Aloe Vera. J Am Podiatr Med Assoc 84:77-81, 1994.

28. DAVIS RH, LEITNER MG, RUSSO JM: Topical anti-inflammatory activity of Aloe Vera as measured by ear swelling. J Am Podiatr Med Assoc 77:610-612, 1987.

29. DAVIS RH, LEITNER MG, RUSSO JM, BYRNE ME: Anti-inflammatory activity of Aloe Vera against a spectrum of irritants. J Am Podiatr Med Assoc 79:263-276, 1989.

30. DAVIS RH, ROSENTHAL KY, CESARIO LR, ROUW GA: Processed Aloe Vera administered topically inhibits inflammation. J Am Podiatr Med Assoc 79:395-397,1989.

31. DAVIS RH, STEWART GJ, BREGMAN PJ: Aloe Vera and the inflamed synovial pouch model. J Am Podiatr Med Assoc 82:140-148, 1992.

32. DIXIT VP, JOSHI S: Effect of Aloe Barbadensis and clofibrate on serum lipids in Triniton- 
induced hyperlipidemia in Presbyter Entellus Entellus Monkeys, Indian J Med Res 78:417421, 1983.

33. GALAL EE, KANDIL A, HEGAZY E, EL GHOROURY M, GOBRAN W: Aloe Vera and gastrointestinal ulceration, J Drug Res Egypt 7:73-77, 1975.

34. GUPTA MB, NATH R, GUPTA GP, BHARGAVA KP: Antiulcer activity of some plant triterpenoids, Indian J Med Res 73:649652, 1981.

35. HIKINO H, HAYASHI T: Hypoglycemic polysaccharides extraction from Aloe species, Jpn Kokai Tokkyo Koho, JP 60,214,741, 28 Oct 1985.
36. HIKINO H, TAKAHASHI M, MURAKAMI M, KONNO C, MIRIN Y, KARIKURA M, HAYASHI T: Isolation and hypoglycemic activity of arborans A and B, glycans of Aloe arborescens var, natalensis leaves, Int J Crude Drug Res 24:183-186, 1986.

37. HUTTER JA, SALMAN M, STAVINOHA WB, SATSANGI N, WILLIAMS RF, STREEPER RT, WEINTRAUB ST: Anti-inflammatory C-glucosyl chromone from Aloe barbadensis, J Natural Prod 59:541-543, 1996.

38. JOSHI S. DIXIT VP: Hypolipidemia effect of Aloe barbadensis (Aloe fraction I) in cholesterol-fed rats. I. Lipid and lipoprotein metabolism, Proc Nat Acad Sci India, Sect B (Biol Sci) 56:339-342, 1986. 